\title{
A new Vitreoscilla filiformis extract grown on spa water-enriched medium activates endogenous cutaneous antioxidant and antimicrobial defenses through a potential Toll-like receptor 2/protein kinase $\mathrm{C}$, zeta transduction pathway
}

This article was published in the following Dove Press journal:

Clinical, Cosmetic and Investigational Dermatology

29 August 2013

Number of times this article has been viewed

\section{Yann F Mahe' \\ Marie-Jesus Perez' \\ Charlotte Tacheau' \\ Chantal Fanchon ${ }^{2}$ \\ Richard Martin ${ }^{3}$ \\ Françoise Rousset \\ Sophie Seite ${ }^{4}$}

'L'Oreal Research and Innovation, Clichy, ${ }^{2}$ ''Oréal Research and Innovation, Chevilly Larue, ' 'L'Oréal Research and Innovation Tours, ${ }^{4}$ La Roche-Posay Pharmaceutical Laboratories, Asnières, France
Correspondence: Sophie Seite La Roche-Posay Pharmaceutical Laboratories, II 0 Avenue Henri Barbusse, 92602 Asnières Cedex, France

Tel +33 । 46886544

Fax +33 | 46882922

Email sophie.seite@loreal.com

\begin{abstract}
Vitreoscilla filiformis (VF) biomass (VFB) has been widely used in cosmetic preparations and shown to modulate the major inducible free-radical scavenger mitochondrial superoxide dismutase in skin cells. By adding La Roche-Posay (LRP) thermal spring water to the VF culture medium, we obtained a biomass (LRP-VFB) with a similar mitochondrial superoxide dismutase activation capacity to VF. Also, the new biomass more powerfully stimulated mRNA expression and antimicrobial peptides in reconstructed epidermis. Interestingly, a predictive computer model that analyzed transducing events within skin epidermal cells suggested that this protective activity may involve the Toll-like receptor 2 /protein kinase $\mathrm{C}$, zeta transduction pathway. Protein kinase C, zeta inhibition was effectively shown to abolish VFB-induced gene stimulation and confirmed this hypothesis. This thus opens new avenues for investigation into the improvement of skin homeostatic defense in relation to the control of its physiological microbiota and innate immunity.
\end{abstract}

Keywords: innate skin defenses, TLR2, PKCz, La Roche-Posay, mitochondrial superoxide dismutase, SOD2

\section{Introduction}

Vitreoscilla filiformis (VF) strain American Type Culture Collection (ATCC) 15551, formerly known as a Beggiatoa sp., is a filamentous bacterium isolated from Lake Erie, an oligotrophic lake in North America. ${ }^{1}$ This bacteria has also been described in hydrothermal vents ${ }^{2}$ and in spa waters known for their beneficial effects on the skin. A continuous process was developed to produce a V. filiformis biomass (VFB), which has been shown to improve skin defense mechanisms.

Both in vitro and in vivo testing have shown that VFB stimulates endogenous antioxidant defenses in skin: in vitro, it stimulated mitochondrial superoxide dismutase 2 (SOD2) activity ${ }^{3}$ both at the mRNA and protein levels, while, in vivo, it decreased the number of ultraviolet-induced sunburn cells in human skin. VFB was thus proposed as an effective anti-aging ingredient for skin improvement and protection from endogenous and exogenous oxidative stress. ${ }^{3}$

This Gram-negative bacterium contains a lipopolysaccharide ${ }^{4}$ (LPS), which reproduces VFB activity once isolated. ${ }^{5}$ Interestingly, it not only stimulates endogenous mitochondrial antioxidant defenses but also endogenous antimicrobial defenses such as 
$\beta$ defensins and S100 calcium-binding protein A7 (S100A7). We hypothesized that the VFB-derived LPS is recognized by Toll-like receptor 2 (TLR2) on skin keratinocytes, which acknowledges the VFB as an exogenous bacterial trigger. This then induces a homeostatic defense reaction and provides a protective biological shield, which reinforces cutaneous resistance and defense mechanisms.

VF belongs to a group of bacterial species that occurs naturally in thermal spa waters (TSW) $)^{1,2}$ and which have been used historically in the management of chronic inflammatory skin diseases. Spa treatments include topical water sprays or jets along with ingestion in the form of a drink. La RochePosay (LRP), France, is a thermal spa particularly known to improve psoriasis and atopic dermatitis. Several publications have highlighted the effects on skin $^{6}$ of its selenium-rich water (LRP-TSW), which particularly protects against the shortand long-term effects of reactive oxygen species induced by ultraviolet radiation as well as inflammation and irritation. A continuous process has previously developed to produce a biomass grown on LRP-TSW-enriched medium (LRP-VFB) and one objective of this study was to compare the biological activities of LRP-VFB and VFB.

Finally, to investigate the possible signaling endogenous pathways linking TLR2 - an identified keratinocyte target of VFB/LRP-VFB involved in the signal transduction of bacterial elements - to the strongly upregulated chemokines chemokine (C-C motif) ligand 20 (CCL20) and interleukin 8 (IL8) that play a major role in the regulation of skin homeostasis, we tested the potential of the PROPAGAPATH ${ }^{7}$ in silico model. PROPAGAPATH ${ }^{7}$ is a dynamic and oriented signaling propagation pathway database, developed by Helios Biosciences (Romainville, France), originally developed, validated, and enriched with immunological and neural cell propagation data. We wanted to evaluate the potency of this model to detect minimal propagation pathways - potentially not already identified by conventional studies - when confronted with data from human skin epidermal keratinocytes.

We used a combination of full genome expression studies and PROPAGAPATH analysis ${ }^{7}$ to understand which endogenous pathways the keratinocytes trigger in response to VF stimulation.

\section{Materials and methods}

\section{In vitro interaction of VFB}

\section{with reconstructed epidermis}

Reconstructed human epidermis (RHE) samples were obtained from SkinEthic Laboratories (Lyon, France). This model is a relevant, reliable, ready-to-use, and robust skin equivalent validated for in vitro alternative testing safety determination and Registration, Evaluation, Authorisation and Restriction of Chemical substances (REACh)-compliant alternatives while respecting ethical human diversity. ${ }^{8}$ They were used at day 17 after the beginning of the reconstruction process. At this time, RHE is histologically similar to the human epidermis with respect to organized keratinocyte layers and progressive cellular differentiation. It contains normal human epidermal keratinocytes (NHEK) cultured on an inert polycarbonate filter at the air-liquid interface, in a chemically defined culture medium. The RHE samples were incubated in a $5 \% \mathrm{CO}_{2}$ atmosphere at $37^{\circ} \mathrm{C}$ overnight. On day 18 , heat-inactivated $\left(120^{\circ} \mathrm{C}, 30\right.$ minutes) $\mathrm{VFB}$, grown on media prepared either with osmosed water (VFB) or LRP-TSW (LRP-VFB), was added at either a concentration of $1 \%$ when applied topically to the RHE samples, or at a concentration of $0.1 \%$ when incubated systemically with the culture medium. All the RHE samples were then incubated in a $5 \% \mathrm{CO}_{2}$ atmosphere at $37^{\circ} \mathrm{C}$ for 18 hours prior to mRNA extraction.

\section{RNA extraction/purification}

The RHE samples were removed from the inserts and total RNA was extracted from each sample using TriPure Isolation Reagent ${ }^{\circledR}$ (Roche Applied Science, Penzberg, Germany) according to the supplier's instructions. The amount and quality of RNA were evaluated using a labon-a-chip Bioanalyzer (Agilent Technologies, Santa Clara, CA, USA). Potential contaminating traces of DNA were removed using the DNA-free ${ }^{\mathrm{TM}}$ system (Life Technologies, Carlsbad, CA, USA).

\section{Reverse transcription and quantitative polymerase chain reactions}

The reverse transcription of mRNA was conducted in presence of oligo-dT and SuperScript ${ }^{\mathbb{}}$ II Reverse Transcriptase (Life Technologies). Quantification of complementary DNA (cDNA) was performed using NanoVue ${ }^{\mathrm{TM}}$ (GE Healthcare, Amersham, UK) and adjustment of cDNA at $10 \mathrm{ng} / \mu \mathrm{L}$.

Polymerase chain reactions (PCRs) were performed in triplicate using the LC480 LightCycler ${ }^{\circledR}$ system (Roche Applied Science) in accordance with the protocol recommended by the supplier. Primer sets were used to amplify the fragments corresponding to the selected markers. In this experiment, the GAPDH (glyceraldehyde 3-phosphate dehydrogenase) gene was used as reference marker (housekeeping gene).

The reaction mix (10 $\mu \mathrm{L}$ final) was added as follows: $2.5 \mu \mathrm{L}$ of cDNA diluted at $10 \mathrm{ng} / \mu \mathrm{L}$, forward and reverse 
corresponding primers, and reagent mix (Roche Applied Science) containing Taq DNA polymerase, SYBR Green I, and $\mathrm{MgCl}_{2}$.

\section{Analysis of quantitative PCR data}

Fluorescence incorporated in amplified DNA was continuously measured during the PCR cycles. This resulted in a "fluorescence intensity" versus "PCR cycle" plot allowing for the evaluation of a relative expression (RE) value for each marker.

The value selected for RE calculations was the "output point" $(\mathrm{Ct})$ of the fluorescence curve. For a given marker, the higher the number of cycles, the lower the mRNA quantity.

The RE value was expressed in arbitrary units according to the formula: $\left(1 / 2^{\text {number of cycles }}\right) \times 10^{6}$.
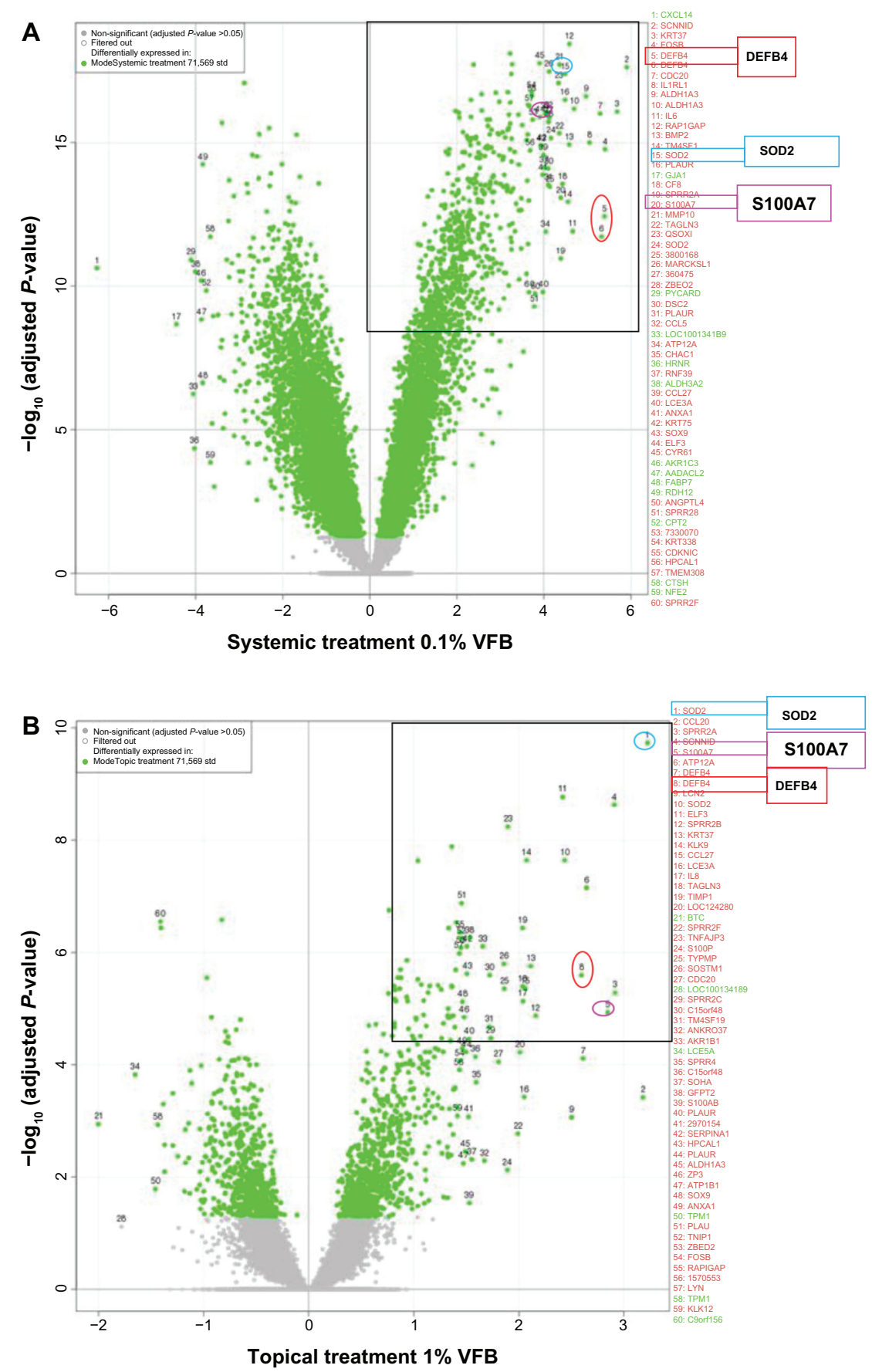

Figure I Full genome expression studies using SkinEthic Laboratories (Lyon, France) reconstructed human epidermises (RHEs) stimulated for I8 hours with Vitreoscilla filiformis biomass (VFB). (A) Systemic incubation of 0.1\% VFB with RHE; (B) topical application of I\% VFB on RHE.

Abbreviations: DEFB4, defensin, beta 4; SI00A7, SI00 calcium-binding protein A7; SOD2, superoxide dismutase 2; std, standard; VFB, Vitreoscilla filiformis biomass. 
Raw data were analyzed with Microsoft Excel ${ }^{\circledR}$ software (Microsoft Corporation, Redmond, WA, USA).

\section{Expression array profiling}

For the expression profiling, biotinylated cRNAs (complementary RNAs) were prepared from $250 \mathrm{ng}$ of total RNA using the Illumina ${ }^{\circledR}$ TotalPrep ${ }^{\text {TM }}$ RNA Amplification Kit (Life Technologies). Then, 750 ng of cRNA per sample was hybridized to the HumanHT-12 v3 Expression BeadChip (Illumina, Inc, San Diego, CA, USA) using the wholegenome gene expression direct hybridization assay protocol recommended by Illumina.

\section{Analysis of expression array}

The gene expression of "treated" RHE was compared with that of "untreated" RHE. One-way analysis of variance was carried out gene by gene and significant differentially expressed genes across the treatments were identified using contrasts based on moderated $t$-statistics. The BenjaminiHochberg correction was applied to the $P$-value for controlling the false discovery rate.

\section{Modelization analysis}

The resulting gene expression data were submitted for analysis and investigation of dynamic signaling pathways to the PROPAGAPATH in silico model. This system is based on the capacity for the scientist to follow the different hypothetical cascading signaling pathways susceptible to rely a chosen entry point - TLR2 in that case - to a deliberated point. We chose IL8 and CCL20 in this experiment.

\section{Effect of PKCz modulation on IL8 and CCL20 expression quantitative real-time $\mathrm{PCR}$ analysis}

NHEK cultures were incubated in a $5 \% \mathrm{CO}_{2}$ atmosphere at $37^{\circ} \mathrm{C}$ for 2 hours with or without $1 \mu \mathrm{M} \mathrm{PKCz}$ pseudosubstrate inhibitor (Calbiochem; EMD Millipore, Billerica, MA, USA). Purified VFB-derived LPS $(10 \mu \mathrm{g} / \mathrm{mL})$ was then added and incubation continued for 6 hours. Keratinocytes were then collected, rinsed with phosphate-buffered saline solution, and freeze-dried at $-80^{\circ} \mathrm{C}$. RNA extraction, purification, and reverse transcription were performed as described.

\section{Results}

Full genome expression following interaction of VFB with RHE

Overall, the data indicated that systemic and topical applications of VFB to RHE resulted in a common modulation of genes, with the systemic contact (Figure 1A) being more potent than the topical route (Figure 1B).

More precisely, these studies showed that VFB significantly induces SOD2 as well as the defensin, beta 4 (DEFB4) (human beta-defensin-2 [HBD2]) and S100A7 antimicrobial defense mRNAs in the reconstructed epidermis (Figure 1).

The right upper corners of Figure $1 \mathrm{~A}$ and $\mathrm{B}$ illustrate the most highly induced genes. DEFB4, SOD2, and S100A7 belong to the top 10 mRNA probes upregulated by VFB. As reported in Table 1, fold changes up to four times $\log 2$ (ie, up to more than 16-fold) were measured when applied directly into the culture medium (systemic incubation). When applied topically to the skin surface, fold changes went up to two times $\log 2$ (ie, close to fourfold).

Western blot analysis of protein synthesis would have provided a useful confirmation, but unfortunately this was not done.

Quantitative real-time PCR analysis showed not only that a classical, systemic incubation with RHE but also a topical application of VFB could significantly engage RHE samples into upregulation of mRNA expression for both endogenous antioxidant $(\mathrm{SOD} 2 \times 3.2)$ and antimicrobial defenses $($ S100A7 $\times 2.8$ and DEFB4 $\times 2.6)$ (Figure 1 and Table 1).

\section{Addition of LRP-TSW to the VFB growth medium}

Table 2 reports comparisons between VFB cultured in the presence of osmosed water (Figure 1 and Table 1) or in the presence of LRP-TSW. While mitochondrial SOD2-mRNA is specifically induced at comparable levels, the transcription of the two antimicrobial peptides HBD2 (DEFB4) and S100A7 was more highly amplified (expressed up to four to six times more) with LRP-VFB than with the original VFB.

NQO1 (NADPH:quinone oxidoreductase 1) and heme oxygenase (decycling) 1 (HMOX1) expression, used as

Table I Calculated fold changes between systemic and topical treatments with Vitreoscilla filiformis biomass (VFB) on a selection of genes

\begin{tabular}{lll}
\hline Gene & $\begin{array}{l}\log _{2} \text { fold change after } \\
\text { systemic incubation with } \\
\text { VFB 0.1\% for 18 hours }\end{array}$ & $\begin{array}{l}\log _{2} \text { fold change after } \\
\text { topical application of } \\
\text { VFB I\% for 18 hours }\end{array}$ \\
\hline SOD2 & $\times 4.5$ & $\times 3.2$ \\
SI00A7 & $\times 4.4$ & $\times 2.8$ \\
DEFB4 & $\times 5.4$ & $\times 2.6$ \\
KRT7 & $\times 5.7$ & $\times 2.1$ \\
\hline
\end{tabular}

Abbreviations: $D E F B$, defensin, beta 4; S100A7, S100 calcium-binding protein A7; SOD2, superoxide dismutase 2; KRT7, keratin 7. 
Table 2 Comparative modulation of mRNA coding for antioxidant and antimicrobial proteins using VFB batches grown on osmosed water medium or on LRP-TSW enriched medium

\begin{tabular}{lll}
\hline & VFB & LRP-VFB \\
\hline SOD2 & 10.02 & 11.67 \\
NQOI & 0.39 & 0.31 \\
HMOXI & 0.75 & 0.75 \\
DEFB4 & 2.64 & 18.77 \\
SIOOA7 & 4.65 & 16.77 \\
\hline
\end{tabular}

Abbreviations: DEFB, defensin, beta 4; SIOOA7, SIO0 calcium-binding protein A7; SOD2, superoxide dismutase 2; HMOXI, heme oxygenase (decycling) I; NQOI, NADPH:quinone oxidoreductase I; LRP, La Roche-Posay; VFB, Vitreoscilla filiformis biomass; TSW, thermal spa waters; mRNA, messenger ribonucleic acid.

negative control targets to ensure the absence of the nuclear factor (erythroid-derived 2)-like 2 activation pathway, were not affected by either form of VFB.

\section{PROPAGAPATH analysis}

We then analyzed the modulation of the full genome microarray data generated on RHE stimulated or not stimulated by VFB by the in silico PROPAGAPATH ${ }^{7}$ dynamic system, with special focus on the possible links between TLR2 stimulation and IL8 and CCL20 modulation.

As shown in Figure 2, the PROPAGAPATH approach defined two possible pathways: the canonical nuclear factor kappa-light-chain-enhancer of activated B cells pathway, which requires at least six and eight steps for IL8 and CCL20, respectively, and also a putative minimal and complementary propagation pathway involving TLR 2 direct $\mathrm{PKCz}$ activation that only requires four and three steps, respectively.

\section{Induction of IL8 and CCL20 are dependent of PKCz activation}

The $\mathrm{PKCz}$ inhibitor markedly altered the expression of the VFB-responsive genes, thus confirming the essential contribution of the TLR2/PKCz pathway in the transduction of VFB into human skin keratinocytes (Figure 3).

$\mathrm{PKCz}$ expression itself was not modulated at the mRNA level, while TLR2, CCL20, and IL8 responded to VFB stimulation. As expected from the modelization program, the use of a PKCz pseudosubstrate inhibitor supressed TLR2, CCL20 and IL8 mRNA induction normally observed in NHEK following VFB stimulation.

\section{Discussion}

TLRs are a key class of pattern-recognition receptors involved in microorganism recognition and induction of antimicrobial peptide responses. ${ }^{9}$ TLR2 activation is known to induce $\beta$-defensin synthesis, while the TLR2-PKCz pathway enhances the tight junction barrier and controls inflammation in various frontier epithelia such as skin, intestinal mucosa, and airway epithelium, ${ }^{10-13}$ which is highly appropriate for maintaining and restoring the integrity and homeostasis of healthy as well as diseased skin. The present study showed that VFB is well recognized by TLR2, induces $\beta$-defensin expression, and stimulates the $\mathrm{PKCz}$ pathway and the mitochondrial antioxidant defense system.

Further, the results of the PROPAGAPATH analysis strongly point at the direct implication of $\mathrm{PKCz}$ in the propagation of innate defenses in human skin keratinocytes in response to VFB. This observation correlates well



Figure 2 PROPAGAPATH (Helios Biosciences, Romainville, France) analysis indicates that PKC $\zeta$ is a potential transducer of the Vitreoscilla filiformis biomass (VFB) activity of Toll-like receptor 2 (TLR2) in skin included in the minimal signaling pathway connecting TLR2 activation to interleukin 8 (IL8) and chemokine (C-C motif) ligand 20 (CCL20) induction.

Abbreviations: FOS, proto oncogene c-Fos; IRAKI, interleukin-I receptor-associated kinase I; MYD88, myeloid differentiation primary response gene (88); NF-KB, nuclear factor kappa-light-chain-enhancer of activated B cells; PMLI, promyelocytic leukemia protein-I; PRKCz, protein kinase C, zeta; PRKR, protein kinase R; SPI, specificity protein I; SRCI, steroid receptor coactivator I; STAT, signal transducer and activator of transcription; STAT3, signal transducer and activator of transcription 3; TIRAP, toll-interleukin I receptor domain containing adaptor protein; TRAF6, tumor necrosis factor receptor-associated factor 6. 


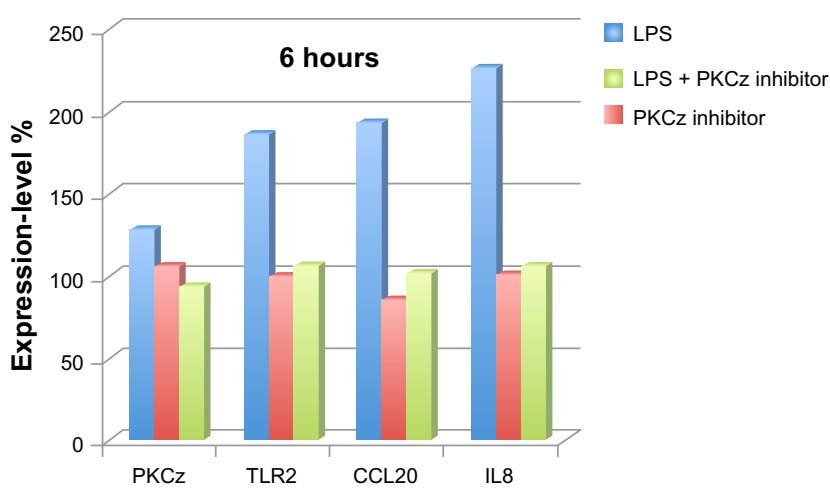

Figure 3 Protein kinase C, zeta (PKCz) transduces Vitreoscilla filiformis biomass (VFB)-derived lipopolysaccharide (LPS) $(10 \mu \mathrm{g} / \mathrm{mL})$ signaling into normal human epidermal keratinocytes. Expression-level fold changes in percentage change (100\% steady-state level; 200\% double expression) for PKCz, TLR2, CCL20, and IL8 in keratinocytes in response to VFB-derived LPS.

Abbreviations: CCL20, chemokine (C-C motif) ligand 20; IL8, interleukin 8; TLR2, Toll-like receptor 2 .

with mechanistic information on epithelial cells. ${ }^{14,15}$ PROPAGAPATH allowed us to identify this pathway for the first time in reconstructed skin, with a minimal number of steps when compared with the canonical myeloid differentiation primary response gene (88), nuclear factor kappa-light-chain-enhancer of activated B cells pathway. The VFB-derived LPS fraction stimulated gene expression and was inhibited with a PKCz inhibitor, suggesting that this specific fraction is indeed a key component of the protective action of VFB on skin, as has already been shown., ${ }^{4,5}$

We also showed that cultivating VF on selenium-rich LRP-TSW produced a biomass with an improved capacity to stimulate innate skin defense biomarkers, namely SOD2, DEFB4, and S100A7.

LRP-VFB is expected to particularly benefit chronic inflammatory skin diseases, such as atopic dermatitis, in which antioxidant, antibacterial, and appropriate immune activities are important therapeutic strategies.

\section{Acknowledgments}

The authors thank Helios Bioscience for the development of and access to the elegant and potent PROPAGAPATH system, and for helpful discussions, and Altrabio SA, Lyon, France, for statistical analysis of the gene expression data.

\section{Disclosure}

All the authors are employees of L'Oréal; they declare no other conflicts of interest in this work.

\section{References}

1. Burton SD, Morita RY. Effect of catalase and cultural conditions on growth of Beggiatoa. J Bacteriol. 1964;88(6):1755-1760.

2. Nelson DC, Wirsen CO, Jannasch HW. Characterization of Large, Autotrophic Beggiatoa spp. Abundant at Hydrothermal Vents of the Guaymas Basin. Appl Environ Microbiol. 1989;55(11):2909-2917.

3. Mahé YF, et al. Induction of the skin endogenous protective mitochondrial MnSOD by Vitreoscilla filiformis extract. Int $J$ Cosmet Sci. 2006;28(4):277-287.

4. Mahé Y, Martin R, inventors; L'Oréal, assignee. Use of a vitreoscilla filiformis lipopolysaccharide fraction as an agent for stimulating the synthesis of anti-microbial skin peptides. European patent EP1974720 A1. March 25, 2008. United States patent US2009035294 A1. February 5, 2009.

5. Martin R, inventor; L'Oréal, assignee. Lipid A-type compound and composition containing it. United States patent US2005118118. June 2, 2005.

6. Seite S. Thermal waters as cosmeceuticals: La Roche-Posay thermal spring water example. Clin Cosmet Invest Derm. 2013;6:1-6.

7. Vujasinovic T, Zampera AS, Jackers P, Sanoudou D, Depaulis A. In silico dynamic molecular interaction networks for the discovery of new therapeutic targets. Curr Pharm Res. 2010;16(20):2241-2251.

8. Alepee N, Grandidier MH, Cotovio J. In vitro Episkin ${ }^{\mathrm{TM}}$ skin corrosion as a reference test method in OECD tg431 for the assessment of skin corrosion in sub-categories 1a, 1b and 1c. Skin Pharmacol Appl Skin Physiol. In press.

9. Kawai T, Akira S. The role of pattern-recognition receptors in innate immunity: update on Toll-like receptors. Nat Immunol. 2010;11(5): 373-384.

10. Kawai K, Shimura H, Minagawa M, Ito A, Tomiyama K, Ito M. Expression of functional Toll-like receptor 2 on human epidermal keratinocytes. J Dermatol Sci. 2002;30(3):185-194.

11. Rakoff-Nahoum S, Paglino J, Eslami-Varzaneh F, Edberg S, Medzhitov R. Recognition of commensal microflora by toll-like receptors is required for intestinal homeostasis. Cell. 2004;118(2):229-241.

12. Hertz CJ, Wu Q, Porter EM, et al. Activation of Toll-like receptor 2 on human tracheobronchial epithelial cells induces the antimicrobial peptide human beta defensin-2. J Immunol. 2003;171(12):6820-6826.

13. Yuki T, Yoshida H, Akazawa Y, Komiya A, Sugiyama Y, Inoue S. Activation of TLR2 enhances tight junction barrier in epidermal keratinocytes. J Immunol. 2011;187(6):3230-3237.

14. Leverence JT, Medhora M, Konduri GG, Sampath V. Lipopolysaccharideinduced cytokine expression in alveolar epithelial cells: role of PKC $\zeta$-mediated p47phox phosphorylation. Chem Biol Interact. 2011; 189(1-2):72-81.

15. Colombo D, Tringali C, Franchini L, Cirillo F, Venerando B. Glycoglycerolipid analogues inhibit PKC translocation to the plasma membrane and downstream signaling pathways in PMA-treated fibroblasts and human glioblastoma cells, U87MG. Eur J Med Chem. 2011;46(5):1827-1834.
Clinical, Cosmetic and Investigational Dermatology

\section{Publish your work in this journal}

Clinical, Cosmetic and Investigational Dermatology is an international, peer-reviewed, open access, online journal that focuses on the latest clinical and experimental research in all aspects of skin disease and cosmetic interventions. All areas of dermatology will be covered; contributions will be welcomed from all clinicians and

\section{Dovepress}

basic science researchers globally. This journal is indexed on CAS The manuscript management system is completely online and includes a very quick and fair peer-review system, which is all easy to use. Visit http://www.dovepress.com/testimonials.php to read real quotes from published authors. 